Marquette University

e-Publications@Marquette

School of Dentistry Faculty Research and

Publications

Dentistry, School of

$5-2017$

\title{
Interleukin-6 Expression in Disc Derangement of Human Temporomandibular Joint and Association with Osteoarthrosis
}

\author{
Flavio Camejo \\ Pontifícia Universidade Católica do Paraná \\ Marina Azevedo \\ Pontificia Universidade Catolica do Parana \\ Viviane Ambros \\ Pontificia Universidade Catolica do Parana \\ Karina Caporal \\ Pontificia Universidade Catolica do Parana \\ Andrea Doetzer \\ Pontifícia Universidade Católica do Paraná
}

See next page for additional authors

Follow this and additional works at: https://epublications.marquette.edu/dentistry_fac

Part of the Dentistry Commons

\section{Recommended Citation}

Camejo, Flavio; Azevedo, Marina; Ambros, Viviane; Caporal, Karina; Doetzer, Andrea; Almeida, Luis Eduardo; Olandoski, Marcia; Noronha, Lucia; and Trevilatto, Paula C., "Interleukin-6 Expression in Disc Derangement of Human Temporomandibular Joint and Association with Osteoarthrosis" (2017). School of Dentistry Faculty Research and Publications. 258.

https://epublications.marquette.edu/dentistry_fac/258 


\section{Authors}

Flavio Camejo, Marina Azevedo, Viviane Ambros, Karina Caporal, Andrea Doetzer, Luis Eduardo Almeida, Marcia Olandoski, Lucia Noronha, and Paula C. Trevilatto 
Marquette University

e-Publications@Marquette

\section{Dentistry Faculty Research and Publications/School of Dentistry}

This paper is NOT THE PUBLISHED VERSION; but the author's final, peer-reviewed manuscript. The published version may be accessed by following the link in the citation below.

Journal of Cranio-Maxillofacial Surgery, Vol. 45, No. 5 (2017): 768-774. DOI. This article is (C) Elsevier and permission has been granted for this version to appear in e-Publications@Marquette. Elsevier does not grant permission for this article to be further copied/distributed or hosted elsewhere without the express permission from Elsevier.

\section{Interleukin-6 expression in disc derangement of human temporomandibular joint and association with osteoarthrosis}

\section{Flaviode Alcântara Camejo}

School of Health and Biosciences, Pontifícia Universidade Católica do Paraná, Curitiba, Brazil Marina Azevedo

School of Health and Biosciences, Pontifícia Universidade Católica do Paraná, Curitiba, Brazil Viviane Ambros

School of Health and Biosciences, Pontifícia Universidade Católica do Paraná, Curitiba, Brazil Karina São Thiago Caporal

School of Health and Biosciences, Pontifícia Universidade Católica do Paraná, Curitiba, Brazil Andrea Duarte Doetzer

School of Health and Biosciences, Pontifícia Universidade Católica do Paraná, Curitiba, Brazil Luis Eduardo Almeida

School of Dentistry, Marquette University, Milwaukee

Marcia Olandoski

School of Health and Biosciences, Pontifícia Universidade Católica do Paraná, Curitiba, Brazil 


\section{Lucia Noronha}

School of Health and Biosciences, Pontifícia Universidade Católica do Paraná, Curitiba, Brazil

Paula Cristina Trevilatto

School of Health and Biosciences, Pontifícia Universidade Católica do Paraná, Curitiba, Brazil

\section{Abstract}

The inflammatory process is a coordinated response that protects host after infection or trauma, involving several molecular reactions. Once the inflammation is closely linked to the process of destruction of the temporomandibular joint, this study aims to examine, by immunohistochemistry, the expression of interleukin-6 (IL-6), an important inflammatory marker, in temporomandibular articular discs of patients with anterior disc displacement with (ADDwR) and without reduction (ADDwoR) and its association with osteoarthrosis $(O A)$. Thirty-eight $(n=38)$ articular discs were divided into two cutoffs: 1) analysis 1: 4 control (acute pathology), 17 ADDwR, 17 ADDwoR; and 2) analysis 2: without OA $(n=21)$ and with $O A(n=17)$. The area of immunostaining was compared statistically between groups $(p<0.05)$. In the disc samples, no significant differences were observed between the groups ADDwR and ADDwoR, and with and without OA, in respect to the expression of IL- 6 by immunohistochemical examination. Future studies should be conducted with a larger sample size, which could clarify the association of the inflammatory mediator IL-6 with temporomandibular joint dysfunction.

\section{Keywords}

Temporomandibular derangement, Osteoarthrosis, IL-6, Temporomandibular disc

\section{Introduction}

A disc derangement is defined as a malposition of the articular disc relative to the condyle and the articular eminence of temporal bone and is usually associated with a reduction or loss of disc mobility. The most common position associated with internal derangement of the temporomandibular joint is anterior disc displacement (pure anterior displacement, anterolateral or anteromedial displacement), and it can be divided into with (ADDwR) or without (ADDwoR) reduction (Chiba et al., 2007, Huddleston Slater et al., 2005, Westesson et al., 1998).

In ADDwR, the articular disc of temporomandibular joint (TMJ) is displaced while the mouth is closed and the teeth are in contact in maximal occlusion and slide into its functional position as the jaw opens (Perez del Palomar and Doblare, 2007, Okeson, 1993). In ADDwoR, the condyle is unable to slide or snap back underneath the disc. The displaced disc thus does not reduce to its position on top of the condyle during the opening movement (Perez del Palomar and Doblare, 2007, Okeson, 1993).

Disc displacement results in stretching and inflammation of the retrodiscal tissue, which often results in internal derangement (ID) of the TMJ, and it is considered to be a risk factor for osteoarthrosis (OA) development, with abnormal remodeling of the condyle and mandibular fossa (Segu et al., 2011, Milam et al., 1998, De Bont and Stengenga, 1993, Isacsson et al., 1986). 
ID is characterized by mechanical interference within the joint, associated with progressive disc displacement secondary to inflammation, trauma (most common etiologic factor) or degeneration, although the real pathogenesis is unclear, and may be associated with genetic predisposition, hormonal influence or microbial invasion (Mcintosh and Dimitroulis, 2012, Dimitroulis, 2011, Dimitroulis, 2011a).

Previous studies have shown the correlation between inflammatory processes and destruction of TMJ components, and interleukin-6 (IL-6) has been pointed out as one of the most important proinflammatory cytokines contributing to the pathogenesis of TMJ ID (Gunson et al., 2012, Sato et al., 2003, Nishimura et al., 2002, Kubota et al., 1998, Fu et al., 1995).

IL-6 is a pleiotropic cytokine produced by several cell types, such as synovial cells, monocytes, macrophages, and fibroblasts, in the TMJ (Nishimoto et al., 2000). It regulates immunological reactions, inflammation, hematopoiesis and oncogenesis (Gunson et al., 2012, Sato et al., 2003), and it mediates the induction of osteoclast progenitor differentiation and osteoclast activity (Fu et al., 1995).

IL-6 is important to the transition from acute to chronic inflammation (Kaplanski et al., 2003), and presents a dual effect; at some levels it acts as pro-inflammatory, whereas in others it can exhibit an anti-inflammatory profile (Yamamoto et al., 2000, Alonzi et al., 1998).

Our objective was to use IL- 6 with proven correlation between the process and destruction of TMJ components, which has not been studied in articular discs. In this study, we tested the hypothesis that this inflammatory marker may be involved in the progression of TMJ ID, as shown by previous research, such as that performed by Sato et al. (2003) using immunohistochemistry, in which where they found expression of IL- 6 in synovial lining cells and in the mononuclear cells infiltrating the periphery of the blood vessels. Therefore, the present investigation was designed to evaluate, through immunohistochemistry, the expression of IL-6 in TMJ articular discs in ADDwR and ADDwoR patients. Moreover, the presence of TMJ OA was also investigated to better understand the relationship between TMJ disc displacement and IL-6 expression.

\section{Materials and methods}

\subsection{Sample selection}

A convenience sample of 38 temporomandibular discs from 25 patients, mean age 37.9 years (range 18-56 years), was recruited for study from the patient pool at the Evangelico School Hospital, Curitiba, southern region of Brazil (Table 1), as approved by the Ethical Committee on Research at Pontifical Catholic University of Paraná, according to Resolution 196/96 of the National Health Council and approved under registration number 104. Subjects were not included if they presented with any of the following: use of orthodontic appliances; chronic use of anti-inflammatory drugs; history of diabetes, hepatitis, HIV infection, or immunosuppressive chemotherapy; history of any disease known to severely compromise immune function; current pregnancy or lactation; dentofacial deformity; major jaw trauma; previous open TMJ surgery; and previous steroid injection in the TMJ. 
Table 1. Baseline clinical characteristics of the study group with and without TMJ dysfunction, associated with Wilkes stage.

\begin{tabular}{|c|c|c|c|c|c|c|c|}
\hline \multirow{2}{*}{ Patient } & \multirow{2}{*}{ Ethnic group } & \multirow{2}{*}{ Gender } & \multirow{2}{*}{ Age (yrs) } & \multirow{2}{*}{ Diagnosis } & \multicolumn{2}{|c|}{ Affected side } & \multirow{2}{*}{ Wilkes stage } \\
\hline & & & & & Right & Left & \\
\hline 1 & Caucasian & $\mathrm{F}$ & 39 & ADDwoR & $x$ & & V \\
\hline 2 & Caucasian & $M$ & 27 & CFx & $x$ & & \\
\hline 3 & Caucasian & $\mathrm{F}$ & 25 & ADDwoR & & $x$ & V \\
\hline 4 & Caucasian & $\mathrm{F}$ & 46 & ADDwoR & & $x$ & V \\
\hline 4 & Caucasian & $\mathrm{F}$ & 46 & ADDwoR & $x$ & & V \\
\hline 5 & Caucasian & $\mathrm{F}$ & 20 & ADDwoR & & $x$ & V \\
\hline 5 & Caucasian & $\mathrm{F}$ & 20 & ADDwoR & $x$ & & $\mathrm{~V}$ \\
\hline 6 & Caucasian & $\mathrm{F}$ & 41 & ADDwR & & $x$ & III \\
\hline 6 & Caucasian & $\mathrm{F}$ & 41 & ADDwR & $x$ & & III \\
\hline 7 & Caucasian & $\mathrm{F}$ & 32 & ADDwR & & $x$ & III \\
\hline 7 & Caucasian & $\mathrm{F}$ & 32 & ADDwR & $x$ & & III \\
\hline 8 & Caucasian & $\mathrm{F}$ & 41 & ADDwoR & & $x$ & V \\
\hline 8 & Caucasian & $\mathrm{F}$ & 41 & ADDwoR & $x$ & & $\mathrm{~V}$ \\
\hline 9 & Caucasian & $\mathrm{F}$ & 26 & ADDwR & & $x$ & III \\
\hline 9 & Caucasian & $\mathrm{F}$ & 26 & ADDwR & $x$ & & III \\
\hline 10 & Caucasian & $\mathrm{F}$ & 28 & ADDwR & & $x$ & III \\
\hline 10 & Caucasian & $\mathrm{F}$ & 28 & ADDwR & $x$ & & III \\
\hline 11 & Caucasian & $\mathrm{F}$ & 36 & ADDwR & & $x$ & III \\
\hline 11 & Caucasian & $\mathrm{F}$ & 36 & ADDwR & $x$ & & III \\
\hline 12 & Caucasian & $\mathrm{F}$ & 18 & ADDwR & & $x$ & III \\
\hline 12 & Caucasian & $\mathrm{F}$ & 18 & ADDwR & $x$ & & III \\
\hline 13 & Caucasian & $\mathrm{F}$ & 38 & ADDwoR & & $x$ & IV \\
\hline 13 & Caucasian & $\mathrm{F}$ & 38 & ADDwoR & $x$ & & IV \\
\hline 14 & Caucasian & $\mathrm{F}$ & 45 & ADDwoR & $x$ & & IV \\
\hline 15 & Caucasian & $\mathrm{F}$ & 51 & ADDwoR & & $x$ & $\mathrm{~V}$ \\
\hline 16 & Caucasian & $\mathrm{F}$ & 33 & ADDwoR & $x$ & & V \\
\hline 16 & Caucasian & $\mathrm{F}$ & 33 & ADDwoR & & $x$ & $\mathrm{~V}$ \\
\hline 17 & Caucasian & $M$ & 22 & CFx & $x$ & & \\
\hline 18 & Caucasian & $\mathrm{F}$ & 35 & ADDwoR & $x$ & & IV \\
\hline 19 & Caucasian & $\mathrm{F}$ & 22 & ADDwR & $x$ & & III \\
\hline 19 & Caucasian & $\mathrm{F}$ & 22 & ADDwR & & $x$ & III \\
\hline 20 & Caucasian & $\mathrm{F}$ & 24 & ADDwR & & $x$ & III \\
\hline 21 & Caucasian & $M$ & 18 & CFx & $x$ & & \\
\hline 22 & Caucasian & $M$ & 37 & CFx & $x$ & & \\
\hline
\end{tabular}




\begin{tabular}{lllllllr} 
Patient & Ethnic group & Gender & Age (yrs) & Diagnosis & \multicolumn{2}{c}{ Affected side } \\
Right & Left & Will \\
23 & Caucasian & F & 23 & ADDwoR & X & IV \\
23 & Caucasian & F & 23 & ADDwoR & & X & IV \\
24 & Caucasian & F & 42 & ADDwR & X & III \\
25 & Caucasian & F & 56 & ADDwR & X & III
\end{tabular}

$A D D W O R$, anterior disc displacement without reduction; $A D D W R$, anterior disc displacement with reduction; $C H$, condylar hyperplasia; $C F x$, condylar fracture; $O A$, osteoarthrosis.

Subjects completed personal medical history questionnaires and, within a protocol approved by an institutional review board, signed a consent form after being advised of the nature of the study. All patients were asked to complete a pain questionnaire, and a clinical examination was performed according to Clinical Practice Guidelines for TMJ surgery of the American Association of Oral Maxillofacial Surgeons. Basically, the clinical examination consisted of palpation of the TMJ region, the occurrence of painful opening/closing mouth, and crepitation. The patients were considered to be affected and treated surgically when presenting painful clinical signs of disc displacement and/or open mouth severe limitation after unsuccessful noninvasive treatment for at least 6 months (also including arthrocentesis in all patients with anterior disc displacement without reduction; $n=11 ; 17$ specimens).

Patients presenting with disc displacement with and without reduction were grouped together for analysis. Patients with acute pathology were used as controls: 4 individuals presented with extraarticular condyle fracture ( $\mathrm{Cfx}$ ) with $90^{\circ}$ displacement and rupture of the internal structures, confirmed by radiographs and $\mathrm{CT}$ scan, who needed to be operated on for the fracture reduction and disc reposition, as follows:

1) Subjects without any signs of disc displacement (acute pathology group; $n=4 ; 4$ specimens); treated by disc repositioning using a Mitek-anchor.

2) Patients presenting anterior disc displacement with reduction (ADDwR; $n=10 ; 17$ specimens); treated by disc repositioning using a Mitek-anchor (15 specimens) or discectomy (2 specimens).

3) Patients presenting with anterior disc displacement without reduction (ADDwoR; $n=11 ; 17$ specimens); treated by discectomy.

Subjects were included first in clinical categories according to the presence or absence of disc displacement, and second according to the presence or absence of osteoarthrosis using the Wilkes classification (Wilkes, 1978).

Patient selection for OA analysis was based on the primary diagnosis of severe TMJ ID. The stages of TMJ ID were classified into mild, intermediate and severe according to Wilkes classification based on clinical, surgical, and pathological stages (Wilkes, 1978). Mild ID (Wilkes stage III) is characterized by simple disc displacement without any morphological alteration of the disc. The intermediate stage (Wilkes stage IV) is characterized by disc displacement and morphological deformity and/or osseous remodeling changes. Severe derangement (Wilkes stage $V$ ) is characterized by perforations of the disc attachments and osseous shift and/or osteoarthritic changes (sclerosis, osteophyte formation, articular surface flattening, depression and/or cystic alterations) (Wilkes, 1978). Patients from the control group 
and those classified as Wilkes III were considered as not presenting with OA, and patients classified as Wilkes IV or $\mathrm{V}$ were included in the OA group, as follows:

1) Patients without OA (acute pathology group + Wilkes stage III; $n=14 ; 21$ specimens);

2) Patient with OA (Wilkes stage IV and V; $\mathrm{n}=11 ; 17$ specimens).

Table 1 shows the baseline characteristics of the sample.

\subsection{Surgical technique}

TMJ surgery was performed according to the technique described by Mehra and Wolford, (2001).

First, the displaced disk is freed by the surgeon entering the upper and lower joint spaces and lysing adhesions. At this point, a small hole is placed through the lateral pole of the condyle from the posterior to the anterior direction. The Mitek bone-cleat introducer is inserted and pushed into the bone, where two small coils unlock and attach the cleat to the inner surface of the condyle cortical bone. A nonresorbable 2-0 or 3-0 suture is placed through the hole and through the disk at the junction of the posterior and intermediate bands, and the disk is tied down to the condylar neck. The deformity of the disk precludes repositioning it into a more normal position, and recontouring the thickened disk with a scalpel is necessary (this scalpeled material constitutes the sample).

This procedure was conducted for all patients with ADDwR (except in two cases) and in the acute pathology group, but in all patients with ADDwoR, where there is major commitment of the TMJ disc, discectomy was performed according to Miloro and Henriksen (2010).

In the CFx patients, the disc displaced by fracture was repositioned. Postsurgical physical therapy was indicated at the discretion of the surgeon.

Histological sections obtained by scalpel of disk excess were prepared for observation of the in situ expression of IL- 6 by immunohistochemistry.

\subsection{Immunohistochemistry}

The TMJ disc sections were deparaffinized with xylol $(2 \times 10 \mathrm{~min})$ and rehydrated with absolute ethyl alcohol $(3 \times 1 \mathrm{~min})$ and $80 \%$ ethyl alcohol $(1 \times 1 \mathrm{~min})$. Endogenous peroxidase activity was quenched by treatment with $\mathrm{H}_{2} \mathrm{O}_{2}$ (5\% in methanol) for 10 min. Target Retrieval Solution ${ }^{\mathrm{TM}}$ (Dako, DK-2600 Glostrup, Denmark) was used prior to slide staining for heat-inducing epitope retrieval (for formalin-fixed, paraffin-embedded material), according to the manufacturer's instructions. The sections were incubated with monoclonal IL-6 antibody (Imuny Biotechnology, Campinas, Brazil), diluted 1:50 in phosphate-buffered saline solution (PBS) and $0.1 \%$ bovine serum albumin (BSA). For negative controls, the primary antibody was not added, and PBS was used instead. The secondary antibody, AdvanceTM (Dako, DK-2600 Glostrup, Denmark), was applied for $30 \mathrm{~min}$, according to the manufacturer's instructions.

The immunoreactions were visualized by incubating the sections using 3,3' diaminobenzidine (DAB) chromogen (OriGene, Rockville, MD) (1 drop in $1 \mathrm{~mL}$ distilled water). The sections were lightly counterstained with Harris hematoxylin for $5 \mathrm{~min}$ and finally mounted. Immunostaining was considered to be specific to IL- 6 because immunoreactivity was not observed in the negative controls.

The color morphometry method was used to analyze the anti-IL-6 immunostained area in the TMJ disc tissue. For this purpose, images of consecutive fields were captured by the 409 objective lens coupled with the BX50 Olympus microscope with the Sony camera Model DXC-107A, and image analysis was 
performed with Image Pro Plus software (Media Cybernetics Inc., Silver Spring, MD, USA). This software allows an observer to select and paint the positive areas to obtain an image model and make the mask for the other stained slides, with an automatic calculation of the area of the positive reaction (Fig. 1). This procedure was performed by a single examiner in a blinded manner. The data were entered into a spreadsheet, and Microsoft Excel (Microsoft Corporation, Redmond, WA, USA) was used for the statistical analysis. The variable area was measured in square micrometers $\left(\mu \mathrm{m}^{2}\right)$ and was obtained with the mean of all positive areas.

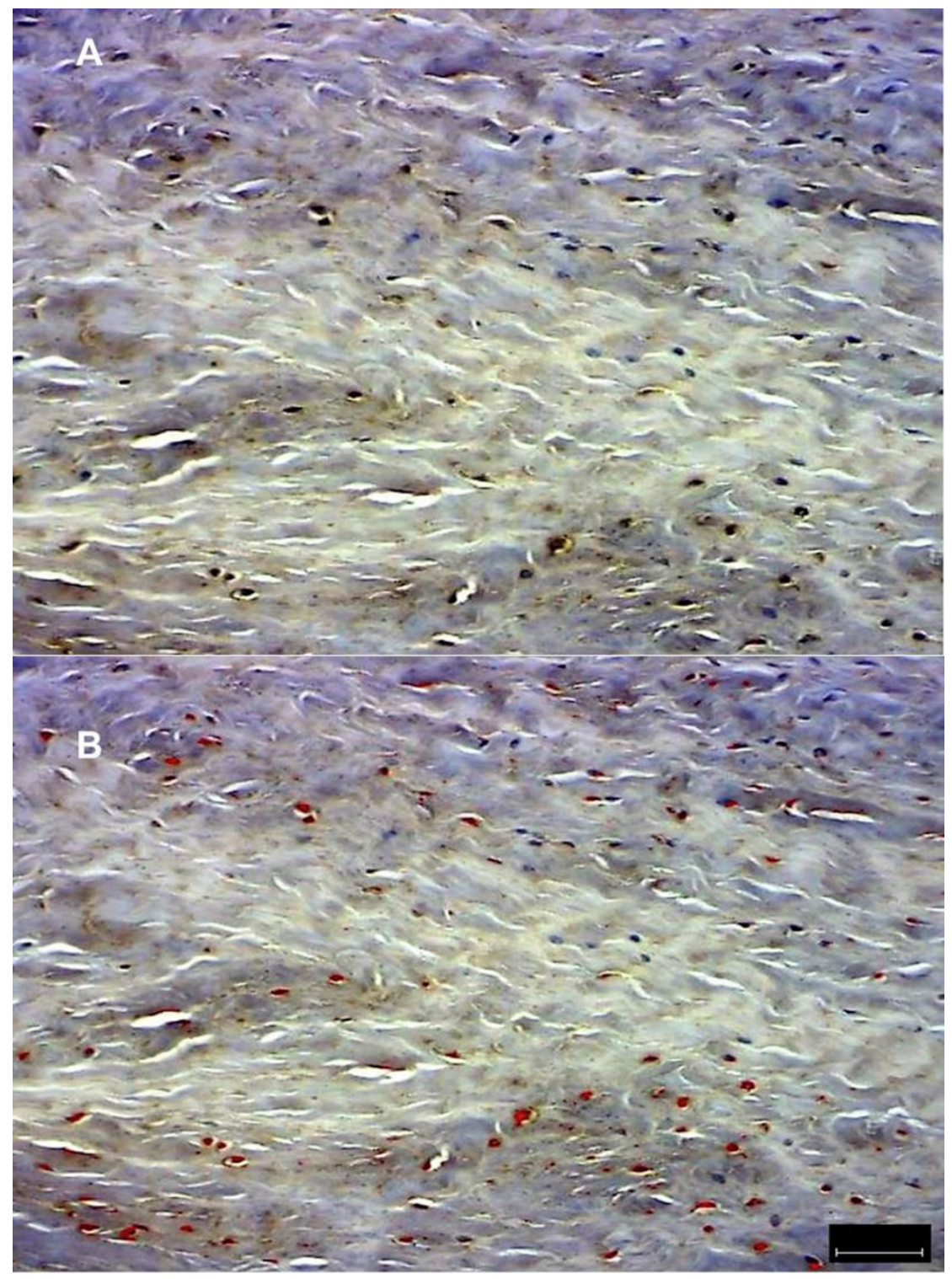

Fig. 1. (A) Photomicrograph showing the positive immunohistochemical reaction for IL-6 in brown, used as the mask. (B) Image Pro Plus ${ }^{\mathrm{TM}}$ analysis of the positive immunohistochemical reaction identified by the software through a sample of brown coloration considered appropriate by the observer; pattern followed for all fields analyzed. The mean of all positive reactions represent the immunohistochemical expression of IL- 6 (measured in $\mu \mathrm{m}^{2}$ ). Scale bar: $100 \mu \mathrm{m}$ (magnification $\left.\times 10\right)$.

\subsection{Statistical analysis}

To compare the groups regarding the area, analysis of variance with a nonparametric Kruskal-Wallis test was used. To compare the control and affected groups, the non-parametric Mann-Whitney test 
was used. To meet the symmetric condition of the variable, data for the area were previously submitted to a logarithmic transformation. A $p$ value $<0.05$ was considered statistically significant for the nonparametric Kruskal-Wallis test, and a $p$ value $<0.025$ for the non-parametric Mann-Whitney test. Data were analyzed with the software IBM SPSS Statistics v.20 (IBM Corporation, Armonk, NY, USA).

\section{Results}

Expression of IL-6 was observed at cytoplasmatic membrane, especially in fibrochondrocytes (Fig. 2, Fig. 3). However, statistically significant differences were not found between TMJ samples of acute pathology, ADDwR and ADDwoR. No association was found between the groups ADDwR and acute pathology, and between the groups ADDwoR and acute pathology. Also, statistically significant differences were not found between the TMJ discs of patients with and without osteoarthrosis.

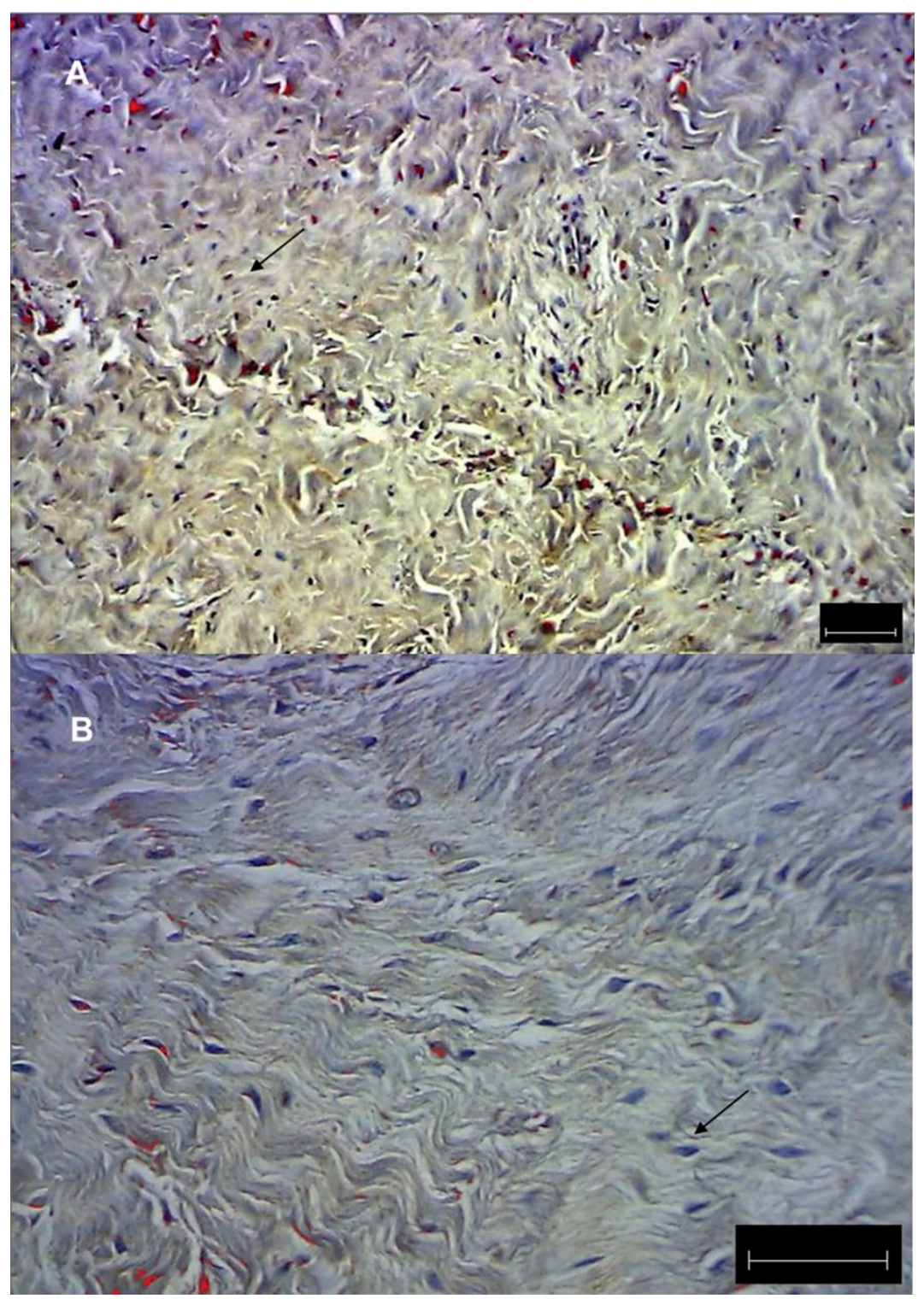

Fig. 2. Immunostaining for IL-6 in the temporomandibular joint disc of patients affected by disc displacement with reduction (ADDwR). (A) Magnification of $\times 10$. Arrow indicates fibrochondrocyte. (B) Magnification of $\times 20$. Arrow indicates fibrochondrocyte. Scale bar: $100 \mu \mathrm{m}$. 


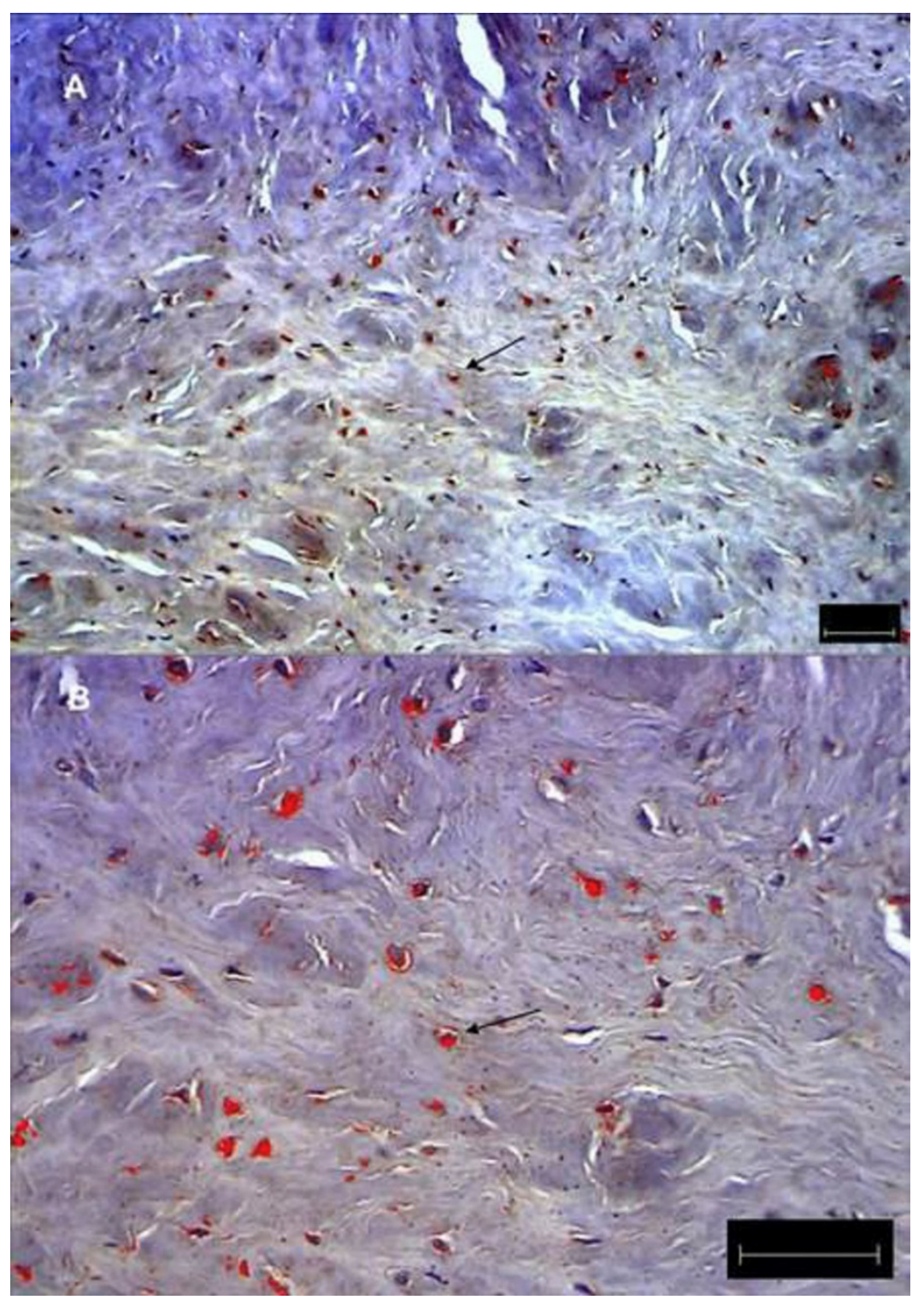

Fig. 3. Immunostaining for IL-6 in the temporomandibular joint disc of patients affected by disc displacement without reduction (ADDwoR). (A) Magnification of $\times 10$. Arrow indicates fibrochondrocyte. (B) Magnification of $\times 20$. Arrow indicates fibrochondrocyte. Scale bar: $100 \mu \mathrm{m}$.

\subsection{Expression of IL-6 in TMJ sample acute pathology $x$ ADDwR $x$ ADDwoR}

Significant differences were not found in the expression of IL-6 in TMJ discs among the three groups for the variable area $(p=0.303)$ (Table 2$)$.

Table 2. IL-6 area of immunostaining $\left(\mu \mathrm{m}^{2}\right)$ in the discs of the control (acute pathology) and study group with and without disc reduction.

\begin{tabular}{|c|c|c|c|c|c|c|c|c|}
\hline Variable & Group & $\mathbf{n}$ & Mean & Median & Minimum & Maximum & Standard deviation & $p$-value* \\
\hline & Control & 4 & 61.8 & 53.8 & 42.4 & 97.1 & 25.6 & \\
\hline \multirow[t]{2}{*}{ Area } & With reduction & 17 & 55.5 & 44.7 & 12.8 & 248.3 & 52.8 & \\
\hline & Without reduction & 17 & 77.1 & 51.6 & 28.8 & 225.1 & 58.7 & 0.303 \\
\hline
\end{tabular}


* Nonparametric Kruskal-Wallis test, $p<0.05$.

3.2. Expression of IL-6 in TMJ sample acute pathology $x$ ADDwR

Significant differences were not found in the expression of IL-6 in TMJ discs between the groups acute pathology and ADDwR for the variable area $(p=0.361)$ (Table 3).

Table 3. IL-6 area of immunostaining $\left(\mu \mathrm{m}^{2}\right)$ in the discs of the control (acute pathology) and group with disc reduction.

\begin{tabular}{|c|c|c|c|c|c|c|c|c|}
\hline Variable & Group & $\mathbf{n}$ & Mean & Median & Minimum & Maximum & Standard deviation & $p$-value* \\
\hline \multirow{2}{*}{ Area } & Control & 4 & 61.8 & 53.8 & 42.4 & 97.1 & 25.6 & \\
\hline & With reduction & 17 & 555 & 44.7 & 12.8 & 248.3 & 52.8 & 0.361 \\
\hline
\end{tabular}

* Nonparametric Mann-Whitney test, $p<0.025$.

\subsection{Expression of IL-6 in TMJ sample acute pathology x ADDwoR}

Significant differences were not found in the expression of IL-6 in TMJ discs between the groups acute pathology and ADDwoR for the variable area $(p=0.965)$ (Table 4).

Table 4. IL-6 area of immunostaining $\left(\mu \mathrm{m}^{2}\right)$ in the discs of the control (acute pathology) and study group without disc reduction.

\begin{tabular}{|c|c|c|c|c|c|c|c|c|}
\hline Variable & Group & n & Mean & Median & Minimum & Maximum & Standard deviation & $p$-value* \\
\hline \multirow{2}{*}{ Area } & Control & 4 & 61.8 & 53.8 & 42.4 & 97.1 & 25.6 & \\
\hline & Without reduction & 17 & 77.1 & 51.6 & 28.8 & 225.1 & 58.7 & 0.965 \\
\hline
\end{tabular}

* Non parametric Mann-Whitney test, $p<0.025$.

3.4. Expression of IL- 6 in TMJ discs of patients with and without osteoarthrosis It was observed that all the patients with ADDwoR presented with OA.

Statistically significant differences were not found in the expression of IL-6 in TMJ discs between the groups with and without OA for the variable area $(p=0.248)$ (Table 5).

Table 5. Differences between groups with and without osteoarthrosis with respect to area of in situ expression $\left(\mu \mathrm{m}^{2}\right)$ of cytokine IL-6.

\begin{tabular}{|c|c|c|c|c|c|c|c|}
\hline Variable & Group & n Mean & Median & Minimum & Maximum & Standard deviation & $p$-value* \\
\hline & Without osteoarthrosis & 2156.7 & 44.7 & 12.8 & 248.3 & 48.3 & \\
\hline-6 are & With osteoarthrosis & 1777.1 & 51.6 & 28.8 & 225.1 & 58.7 & 0.248 \\
\hline
\end{tabular}




\section{Discussion}

For the past few years, many papers have been published showing the participation of various markers and distinct pathways in the progression of TMJ ID using TMJ discs and synovial fluid (Kubota et al., 1998, De Alcantara Camejo et al., 2014, Loreto et al., 2013, Wake et al., 2013, Kim et al., 2012, Leonardi et al., 2011, Loreto et al., 2011, Leonardi et al., 2010a, Leonardi et al., 2010, Loreto et al., 2010, Vernal et al., 2008, Sato et al., 2003).

Sato et al., in 2003, performed a study using also an immuno-histochemical technique for the examination of synovial tissue from 46 temporomandibular joints with internal derangement in 44 patients. As controls, they examined synovial tissue specimens from 7 joints with habitual dislocation without pain. In synovial tissues from 21 of the 46 joints with internal derangement, IL- 6 was expressed in the synovial lining cells and in the mononuclear cells infiltrating the periphery of the blood vessels. The density of IL-6-stained cells in specimens with ID correlated significantly with the grade of joint effusion shown by magnetic resonance imaging ( $p=0.01, r=0.32)$.

In diseases of the TMJ, the synovium and articular cartilage produced various mediators that have the potential to induce chondrocyte death through necrosis or apoptosis (Houston and O'connell, 2004, Gu et al., 2002). De Alcantara Camejo et al., (2014) showed a higher expression of FasL, an apoptotic marker, in TMJ discs with reduction when compared with discs without reduction (in which a less intense inflammatory process and more mechanical stress may coexist), and a lower expression of this marker in the discs of patients with osteoarthrosis. This suggests that apoptosis may protect against TMJ disorder progression, and reinforces that necrosis should be the main means of cell death in OA fibrochondrocytes.

One of the most important and studied pathways in the progression of TMJ ID is inflammation. The clinical symptoms of temporomandibular disorders involve pain and dysfunction during mandibular movement, which are basically symptoms of inflammation. Inflammatory changes in the TMJ modify physical and functional properties, first reversibly and finally irreversibly, reducing its ability to withstand pressure and compression forces (Israel et al., 1991). Loreto et al. (2013). showed that MMP-7 and MMP-9 are expressed in arthritic joints, and provided evidence of a role for those MMPs in disc damage in TMJ ID, with higher expression detected in the posterior band rather than in the anterior and intermediate bands of ADDwR and ADDwoR discs. Cytokines have emerged as the master controllers of hard tissue degradation in human joint tissue in both osteoarthritis and inflammatory arthritis models (Nishimura et al., 2002, Fu et al., 1995). Kim et al. (2012), using TMJ synovial fluid, demonstrated a higher expression of IL-1 $\beta$, IL- 6 and granulocyte macrophage colony stimulating factor (GM-CSF) in patients with temporomandibular dysfunction.

IL-6 is one of the main cytokines involved in this process. It is produced by many kinds of cells, such as monocytes/macrophages, T cells, fibroblasts, and endothelial cells, and promotes T-cell and B-cell differentiation, usually elevated in sepsis or aseptic inflammation (such as rheumatoid arthritis) (Lipsky, 2006).

IL-6 may have a role in both acute and chronic phases of inflammation. Acute inflammation is a beneficial and limited response, particularly during infectious conditions, whereas chronic inflammation is a persistent phenomenon that can lead to tissue damage and to angiogenesis involving the synovium, articular cartilage, and bone. One characteristic of acute inflammation is that initially, the infiltration of leukocytes is predominantly neutrophilic, but after $24-48 \mathrm{~h}$, monocytic cells 
predominate. In contrast, chronic inflammation is associated histologically with the presence of mononuclear cells such as lymphocytes and macrophages.

IL-6 causes not only the acute phase reaction, but also the development of specific cellular and immune humoral responses, including the final stage of B-cell differentiation, immunoglobulin secretion and activation of $T$ cells. The main mechanism of acute inflammation to chronic inflammation is the recruitment of monocytes to the inflammation area, which explains the importance of IL-6 in this process. In models of chronic inflammatory diseases, such as arthritis induced by collagen, IL-6 is proinflammatory, whereas in models of acute inflammation, IL-6 exhibits an anti-inflammatory profile (Kaplanski et al., 2003, Yamamoto et al., 2000, Alonzi et al., 1998).

Since the inflammatory process influences the progression of ID, this study was designed to test the hypothesis that IL-6 may be more highly expressed in the TMJ disc in cases of ADDwR and ADDwoR, and not only in the synovial fluid, as already extensively described in the literature (De Alcantara Camejo et al., 2014, Loreto et al., 2013, Kim et al., 2012, Sato et al., 2003).

Although Brazil has a mixed population, our study population was formed only by individuals of Caucasian/white ethnicity, which can be explained because this study was carried out in the southern region of Brazil, where the majority of the population is descended from Europeans, especially from Poles, Ukrainians, Germans and Italians. Discopexy with disc repositioning was performed using a Mitek anchor in all patients in the acute pathology group and in most cases of ADDwR. In 2 cases ( 2 samples from 2 different patients), the TMJ disc was highly compromised and discectomy was performed. Due to the degenerative impairment found in all cases of ADDwoR, discectomy was performed and physiotherapy instituted.

Significant differences were not observed between the groups by acute pathology and with and without anterior disc reduction of the articular disc with respect to the variable area for the expression of IL-6 as measured by immunohistochemical examination. It was observed, however, that although an increase was not statistically significant, a higher IL-6 expression was found in the group with the more extreme phenotype (without reduction), in which continuous chronic inflammation had jeopardized the tissue.

The presence of TMJ OA was also investigated to better understand the relationship between TMJ disC displacement and IL-6 expression. OA is a focal degenerative disorder that primarily affects the articular cartilage and subchondral bone of synovial joints such as TMJ, which ultimately relies on osteoclastic activity (Vernal et al., 2008, Dimitroulis, 2005). There is consensus that TMJ disc displacement and OA often occur concomitantly, and that disc displacement may promote OA (Dimitroulis, 2005, Nagai et al., 2003, De Bont et al., 1997). In our study no significant differences were observed between the groups with and without osteoarthrosis with respect to the variable area for the expression of IL- 6 as measured by immunohistochemical examination, but the results suggested a higher expression in the group with osteoarthritis, the last stage of a chronic inflammatory destructive process. This may represent a frustrated attempt to control disease progression.

The lack of difference between the groups in this study does not mean, however, that the levels of IL- 6 are not really increased in cases of disc displacement without reduction, and with osteoarthrosis.

This study has some limitations, such as the small sample size together with the control group, which includes patients with condyle fracture (acute pathology). Although the sample size is extremely reduced, it is noteworthy that only about $5 \%$ of people worldwide present with a functional or 
pathological TMJ problem that is amenable to surgery (Dimitroulis, 2011, Dimitroulis, 2011a) and, for ethical reasons, it is not possible to retrieve healthy retrodiscal tissue from normal patients to serve as controls. In our results, an inflammatory cellular infiltrate was seldom observed in this group with acute pathology used as controls (patients with mandibular condyle fractures) associated with the degree of vascularization. Although showing normal characteristics and without a previous history of pain and/or functional limitation, this findings perhaps was due to the fact that the disc is a continually remodeling anatomic structure in a progressive process of healing in response to micro or macro trauma during functional biomechanical movements. Moreover, our sample may be one of largest samples in vivo composed of articular discs. Besides, there was a very high variability in IL-6 expression inside each group, caused, for example by age, individual response, and time of disease progression, which might have contributed to this finding.

\section{Conclusion}

The statistically significant differences observed in the analysis of this study, suggest the importance of IL-6 expression in temporomandibular articular discs on the progression of TMJ ID. A higher IL-6 expression was found in the group with a more extreme phenotype (without reduction), in which continuous chronic inflammation has jeopardized the tissue; and a higher expression in the group with osteoarthritis (the last stage of a chronic inflammatory destructive process), which may represent a frustrated attempt to control disease progression.

Future studies should be conducted with a larger in vivo sample size, with a varied ethnic group, and with other inflammatory markers, which could clarify the impact of inflammation on the progression of TMJ ID.

\section{Disclosures}

Authors have nothing to disclosures.

\section{Acknowledgements}

The first author would like to thank Pontifical Catholic University of Paraná (PUCPR) for the PhD scholarship provided.

\section{References}

Alonzi et al., 1998 T. Alonzi, E. Fattori, D. Lazzaro, P. Costa, L. Probert, G. Kollias, et al. Interleukin-6 is required for the development of collagen-induced arthritis. J Exp Med, 187 (1998), pp. 461-468.

Chiba et al., 2007 M. Chiba, N. Watanabe, S. Echigo. Longitudinal MRI follow-up of non-reducible posterior disc displacement accompanied by bone marrow oedema in the mandibular condyle. Dentomaxillofac Radiol, 36 (2007), pp. 304-307.

De Alcantara Camejo et al., 2014 F. De Alcantara Camejo, L.E. Almeida, A.D. Doetzer, K.S. Caporal, V. Ambros, M. Azevedo, et al. Fasl expression in articular discs of human temporomandibular joint and association with osteoarthrosis. J Oral Pathol Med, 43 (1) (2014), pp. 69-75.

De Bont and Stengenga, 1993 L.G.M. De Bont, B. Stengenga. Pathology of temporomandibular joint internal derangement and osteoarthrosis. Int J Oral Maxillofac Surg, 22 (1993), pp. 71-74. 
De Bont et al., 1997 L.G.M. De Bont, L.C. Dijkgraaf, B. Stegenga. Epidemiology and natural progression of articular temporomandibular disorders. Oral Surg Oral Med Oral Pathol Oral Radiol Endod, 83 (1997), pp. 72-76.

Dimitroulis, 2011 G. Dimitroulis. A critical review of interpositional grafts following temporomandibular joint discectomy with an overview of the dermis-fat graft. Int J Oral Maxillofac Surg, 40 (6) (2011), pp. 561-568.

Dimitroulis, 2011a G. DimitroulisTemporomandibular joint surgery: what does it mean to the dental practitioner? Aust Dent J, 56 (3) (2011), pp. 257-264.

Dimitroulis, 2005 G. Dimitroulis. The role of surgery in the management of disorders of the temporomandibular joint: a critical review of the literature. Part 1. Int J Oral Maxillofac Surg, 34 (2005), pp. 107-113.

Fu et al., 1995 K. Fu, X. Ma, Z. Zhang, X. Pang, W. ChenInterleukin-6 in synovial fluid and HLADR expression in synovium from patients with temporomandibular disorders. J Orofac Pain, 9 (1995), pp. 131-137.

Gu et al., 2002 Z. Gu, T. Shibata, Z. Cao, J. Feng, J. Hu. Chondrocyte apoptosis in temporomandibular joints with disc displacement. J Oral Maxillofac Surg, 60 (2002), pp. 1026-1031.

Gunson et al., 2012 M.J. Gunson, G.W. Arnett, S.B. MilamPathophysiology and pharmacologic control of osseous mandibular condylar resorption. J Oral Maxillofac Surg, 70 (8) (2012), pp. 19181934.

Houston and O'connell, 2004 A. Houston, J. O'connell. The Fas signalling pathway and its role in the pathogenesis of cancer. Curr Opin Pharmacol, 4 (2004), pp. 321-326.

Huddleston Slater et al., 2005 J.J. Huddleston Slater, F. Lobbezoo, N. Hofman, M. Naeijen. Case report of a posterior disc displacement without and with reduction. J Orofac Pain, 19 (2005), pp. 337342.

Isacsson et al., 1986 G. Isacsson, A. Isberg, A.S. Johansson, O. LarsonInternal derangement of the temporomandibular joint: radiographic and histologic changes associated with severe pain. J Oral Maxillofac Surg, 44 (1986), pp. 771-778.

Israel et al., 1991 H.A. Israel, F. Saed-Nejad, A. Ratcliffe. Early diagnosis of osteoarthrosis of the temporomandibular joint: correlation between arthroscopic diagnosis and keratan sulfate levels in the synovial fluid. J Oral Maxillofac Surg, 49 (7) (1991), pp. 708-711.

Kaplanski et al., 2003 G. Kaplanski, V. Marin, F. Montero-Julian, A. Mantovani, C. Farnarier. IL-6: a regulator of the transition from neutrophil to monocyte recruitment during inflammation. Trends Immunol, 24 (2003), pp. 25-29.

Kim et al., 2012 Y.K. Kim, S.G. Kim, B.S. Kim, J.Y. Lee, P.Y. Yun, J.H. Bae, et al. Analysis of the cytokine profiles of the synovial fluid in a normal temporomandibular joint: preliminary study.

J Craniomaxillofac Surg, 40 (8) (2012), pp. e337-e341.

Kubota et al., 1998 E. Kubota, T. Kubota, J. Matsumoto, T. Shibata, K. Murakami. Synovial fluid cytokines and proteinases as markers of temporomandibular joint disease. J Oral Maxillofac Surg, 56 (1998), pp. 192-198.

Leonardi et al., 2011 R. Leonardi, L.E. Almeida, M. Rusu, E. Sicurezza, G. Palazzo, C. Loreto. Tumor necrosis factor-related apoptosis-inducing ligand expression correlates to temporomandibular joint disk degeneration. J Craniofac Surg, 22 (2011), pp. 504-508.

Leonardi et al., 2010 R. Leonardi, M.R. Migliore, L.E. Almeida, P.C. Trevilatto, C. Loreto. Limited fatty infiltration due to apoptosis in human degenerated temporomandibular joint disks: an immunohistochemical study. J Craniofac Surg, 21 (2010), pp. 1508-1511. 
Leonardi et al., 2010a R. Leonardi, L.E. Almeida, P. Trevilatto, C. Loreto. Occurrence and regional distribution of TRAIL and DR5 on temporomandibular joint discs: comparison of disc derangement with and without reduction. Oral Surg Oral Med Oral Pathol Oral Radiol Endod, 109 (2010), pp. 244-251.

Lipsky, 2006 P.E. Lipsky. Interleukin-6 and rheumatic diseases. Arthritis Res Ther, 8 (Suppl. 2) (2006), p. S4.

Loreto et al., 2011 C. Loreto, L.E. Almeida, P. Trevilatto, R. Leonardi. Apoptosis in displaced temporomandibular joint disc with and without reduction: an immunohistochemical study. J Oral Pathol Med, 40 (2011), pp. 103-110.

Loreto et al., 2010 C. Loreto, L.E. Almeida, M.R. Migliore, M. Catalbiano, R. Leonardi. TRAIL, DR5 and caspase 3-dependent apoptosis in vessels of diseased human temporomandibular joint disc. An immunohistochemical study. Eur J Histochem, 54 (2010), p. e40.

Loreto et al., 2013 C. Loreto, R. Leonardi, G. Musumeci, G. Pannone, S. Castorina. An ex vivo study on immunohistochemical localization of MMP-7 and MMP-9 in temporomandibular joint discs with internal derangement. Eur J Histochem, 57 (2) (2013), p. e12.

Mcintosh and Dimitroulis, 2012 M. Mcintosh, G. Dimitroulis. Microbiological investigation of retrodiscal tissues from patients with advanced internal derangement of the temporomandibular joint. Int J Oral Maxillofac Surg, 41 (3) (2012), pp. 313-316.

Mehra and Wolford, 2001 P. Mehra, L.M. Wolford. Use of the Mitek anchor in temporomandibular joint discrepositioning surgery. Proc Bayl Univ Med Cent, 14 (2001), pp. 22-26.

Milam et al., 1998 S.B. Milam, G. Zardeneta, J.P. Schmitz. Oxidative stress and degenerative temporomandibular joint disease: a proposed hypothesis. J Oral Maxillofac Surg, 56 (1998), pp. 214-223.

Miloro and Henriksen, 2010 M. Miloro, B. Henriksen. Discectomy as the primary surgical option for internal derangement of the temporomandibular joint. J Oral Maxillofac Surg, 68 (4) (2010), pp. 782-789.

Nagai et al., 2003 H. Nagai, H. Kumamoto, M. Fukuda, T. Takahashi. Inducible nitric oxide synthase and apoptosis-related factors in the synovial tissues of in temporomandibular joints with internal derangement and osteoarthritis. J Oral Maxillofac Surg, 61 (2003), pp. 801-807.

Nishimoto et al., 2000 N. Nishimoto, T. Kishimoto, K. YoshizakiAnti-interleukin 6 receptor antibody treatment in rheumatic disease. Ann Rheum Dis, 59 (Suppl. 1) (2000), pp. i21-i27.

Nishimura et al., 2002 M. Nishimura, N. Segami, K. Kaneyama, T. Suzuki, M. Miyamaru. Proinflammatory cytokines and arthroscopic findings of patients with internal derangement and osteoarthritis of the temporomandibular joint. Br J Oral Maxillofac Surg, 40 (2002), pp. 68-71.

Okeson, 1993 J.P. Okeson. Management of temporomandibular disorders and occlusion. 294, MosbyYear Book, Inc, St Louis, MO (1993), pp. 409-477.

Perez del Palomar and Doblare, 2007 D.P. Perez del Palomar, M. Doblare. An accurate simulation model of anteriorly displaced TMJ discs with and without reduction. Med Eng Phys, 29 (2007), pp. 216-226.

Sato et al., 2003 J. Sato, N. Segami, M. Nishimura, N. Demura, H. Yoshimura, Y. Yoshitake, et al. Expression of interleukin 6 in synovial tissues in patients with internal derangement of the temporomandibular joint. Br J Oral Maxillofac Surg, 41 (2) (2003), pp. 95-101.

Segu et al., 2011 M. Segu, L. Politi, S. Galioto, V. Collesano. Histological and functional changes in retrodiscal tissue following anterior articular disc displacement in the rabbit: review of the literature. Minerva Stomatol, 60 (7-8) (2011), pp. 349-358. 
Vernal et al., 2008 R. Vernal, E. Velasquez, J. Gamonal, J.A. Garcia-Sanz, A. Silva, M. Sanz. Expression of proinflammatory cytokines in osteoarthritis of the temporomandibular joint. Arch Oral Biol, 53 (10) (2008), pp. 910-915.

Wake et al., 2013 M. Wake, Y. Hamada, K. Kumagai, N. Tanaka, Y. Ikeda, Y. Nakatani, et al. Upregulation of interleukin- 6 and vascular endothelial growth factor-A in the synovial fluid of temporomandibular joints affected by synovial chondromatosis. Br J Oral Maxillofac Surg, 51 (2) (2013), pp. 164-169.

Westesson et al., 1998 P.I. Westesson, T.A. Larheim, H. TanakaPosterior disc displacement in the temporomandibular joint. J Oral Maxillofac Surg, 56 (1998), pp. 1266-1273.

Wilkes, 1978 C. WilkesArthrography of the temporomandibular joint inpatients with the TMJ paindysfunction syndrome. Minn Med, 61 (1978), pp. 645-652.

Yamamoto et al., 2000 M. Yamamoto, K. Yoshizaki, T. Kishimoto, H. Ito. IL-6 is required for the development of Th-1 cell-mediated murine colitis. J Immunol, 164 (2000), pp. 4878-4882. 